\title{
Gas Supersaturation Thresholds for Spontaneous Cavitation in Water with Gas Equilibration Pressures up to 570 atm $^{1}$
}

\author{
Wayne A. Gerth and Edvard A. Hemmingsen * \\ Physiological Research Laboratory \\ Scripps Institution of Oceanography University of California, San Diego \\ La Jolla, California 92093 \\ (Z. Naturforsch. 31 a, 1711-1716 [1976] ; received October 5, 1976)
}

\begin{abstract}
Supersaturations for the onset of cavitation in water were determined for various gases. At ambient pressure, the threshold supersaturations (in atm) required for profuse cavitation to initiate both in bulk water and at the glass-water interface were $\mathrm{CH}_{4}, 120 ; \mathrm{Ar}, 160 ; \mathrm{N}_{2}, 190 ; \mathrm{He}, 360$. Exposure of the water and its containing surfaces to hydrostatic pressures up to $1100 \mathrm{~atm}$ prior to equilibration with gas had no detectable effect on these threshold values. This indicates that preformed gas nuclei are not significantly involved. Cavitation at the glass-water interface was investigated for $\mathrm{CH}_{4}, \mathrm{Ar}$ and $\mathrm{N}_{2}$ saturations up to $570 \mathrm{~atm}$. For 320 atm saturations, the threshold supersaturation was about one-half that for decompression directly to ambient pressure, and for $550 \mathrm{~atm}$ it was about one-third. These results indicate that the dissolved gas concentration is a critical factor limiting the spontaneous nucleation of bubbles in gas supersaturated liquids.
\end{abstract}

\section{Introduction}

Water that contains large gas supersaturations can remain stable with the absence of cavitation ${ }^{2,3}$. However, as the gas supersaturation in the water is increased, the system eventually fails to tolerate the supersaturation and bursts profusely with the formation of bubbles. Hemmingsen ${ }^{3}$ reported the largest observed gas supersaturation stabilities of water and found that the minimum supersaturation required for cavitation inception, in the apparent absence of pre-formed nuclei, varied substantially with different gases in solution. This threshold supersaturation generally increased with decreasing gas solubility, suggesting that the total concentration of gas in solution is important in determining the gas supersaturation stability of liquids.

Our efforts to further characterize cavitation nucleation phenomena required examination of this concentration effect in some detail. An experimental system was developed in which wide ranges of gas pressure could be used to vary the dissolved gas concentrations, necessitating the minimization of interference by foreign gas nuclei whose persistence in the water and on container surfaces could vary as a function of pressure. This was accomplished by applying large hydrostatic pressures to the water and the container surfaces prior to solution of the gas,

\footnotetext{
* Reprint requests to Dr. Edvard A. Hemmingsen, A-004 Physiological Research Laboratory, Scripps Institution of Oceanography, La Jolla, CA 92093, USA.
}

thereby forcing the resolution of such dissolvable nuclei ${ }^{4}$.

The results reported here bear directly upon the understanding of the gas supersaturated metastable condition in pure liquids, the factors which limit it, and the characteristics of its degeneration via the nucleation and growth of bubbles. These results also have implications for the understanding of the formation of bubbles in organisms with gas supersaturated tissues, such as occurs in conjunction with decompression sickness in aviators and divers ${ }^{5,6}$ and in tumors supersaturated with oxygen from injection of hydrogen peroxide as an adjuvant to radiation therapy ${ }^{7}$.

\section{Methods}

The apparatus (Fig. 1) was designed to first hydrostatically pressurize water, then to equilibrate a small portion under high gas pressure, and finally, to transfer it under pressure into an observation capillary where it could be observed with a binocular microscope $(15 \times)$ during decompression. All chambers and tubing were made of stainless steel. Chamber A contained a $2.5 \mathrm{~cm}$ thick quartz viewing port and a plexiglass lighting port. The pyrex observation capillary $(0.011 \mathrm{~cm}$ I.D.) was placed inside this chamber to enable high pressurization of the water and its container surfaces. The capillary had a volume of $0.07 \mathrm{ml}$ and was connected with a plastic sleeve to a steel tube $(0.025 \mathrm{~cm}$ I.D.) protruding into the chamber. The pyrex sample bowl 




Fig. 1. Schematic diagram of apparatus with enlarged illustration of Chamber A showing arrangement of observation capillary, venting device, and sample bowl.

had a volume of $1.5 \mathrm{ml}$ and was secured in the chamber with a plexiglass retainer. The capillary venting device on top of the chamber had a Viton rubber seal retained by a piston and screw assembly. Chamber B, with a movable "O" ring sealed plexiglass piston, could be filled below the piston with water, gas equilibrated with only atmospheric pressure.

Each of the gases used: methane, argon, nitrogen and helium; had a purity greater than $99.9 \%$. Commercial bottled distilled water was used without further treatment. Experiments were conducted at room temperature which usually ranged from $19^{\circ} \mathrm{C}$ to $21{ }^{\circ} \mathrm{C}$. The capillary was cleaned in concentrated sodium dichromate - sulfuric acid solution and rinsed with distilled water before installation in the chamber and periodically during the investigation. Capillaries found to have obvious flaws adversely affecting the gas supersaturation stability of the water were replaced.

Chamber A was filled completely with water with the hydraulic pump at the start of each experiment. Hydrostatic pressure from $935 \mathrm{~atm}$ to $1100 \mathrm{~atm}$ was then applied for a period of at least 15 minutes, after which water was slowly forced out the bottom inlet by gas pressure. The water retained in the sample bowl was equilibrated with gas at the desired pressure for at least one hour while being stirred at $60 \mathrm{rpm}$ with a small Teflon-coated stirring bar. After equilibration, the water was slowly forced into the capillary from the sample bowl by slightly loosening the inner screw which maintained the seal over the capillary venting device until the sample bowl was nearly emptied. The gas remaining in chamber A was replaced isobarically with water from chamber $B$ to prevent fogging during decompression. Chamber A was decompressed immediately thereafter while visually observing the capillary for the appearance of bubbles. In one series of experiments, the chamber was rapidly $(2-3$ seconds $)$ decompressed directly to ambient pressure. In subsequent experiments, gradual decompressions were used and the pressures at which bubbles were first visible in the capillary were noted. The supersaturation tension was then defined as the difference between the equilibration pressure and the latter decompression pressure.

Cavitation was characterized as follows: no cavitation - no bubbles formed in the capillary within approximately 2 minutes of decompression; light cavitation - relatively few $(1-20)$ bubbles formed, leaving most of the water in the capillary bubble free; massive cavitation - bubbles formed effervescently. Moderate cavitation characterizes those intermediate cases in which many bubbles formed throughout the capillary, but in which the cavitation was judged to fall short of effervescence.

\section{Results}

The cavitation behavior of hydrostatically prepressurized water, gas supersaturated by decompression directly to ambient pressure, is shown in Figure 2. Also shown are experiments in which no special hydrostatic pre-treatment of the water was used. Absence of cavitation with supersaturation tensions of $100 \mathrm{~atm}$ or more was possible with each of the gases. The greatest supersaturation tension with absence of cavitation was obtained with $\mathrm{He}$ at $320 \mathrm{~atm}$. With increasing gas supersaturations, bubbles began to form in increasing numbers. The onset of cavitation occurred at different supersaturations for each of the gases. Light to moderate cavitation always appeared to initiate at or near the glasswater interface. Massive cavitation, however, was caused by widespread fracturing of the water both at the glass-water interface and uniformly in the bulk water, and was never observed below a certain supersaturation tension which was specific for each gas. The supersaturation threshold for cavitation in 
bulk water, i. e. the bulk threshold, required in excess of ambient pressure was $120 \mathrm{~atm}$ for $\mathrm{CH}_{4}$, $160 \mathrm{~atm}$ for Ar, $190 \mathrm{~atm}$ for $\mathrm{N}_{2}$, and approximately $360 \mathrm{~atm}$ for $\mathrm{He}$. The bulk threshold for $\mathrm{He}$ was less well defined than for the other gases due to the rapid expansion of bubbles at the glass-water interface. Comparison with data obtained without hydrostatic pre-pressurization of the water (also see Ref. ${ }^{3}$ ) indicates that pre-pressurization had no noticeable effect on any cavitation character except for the supersaturation required for inception of very light cavitation, which in only a few cases was slightly decreased.

Bubbles formed well before ambient pressure had been reached during decompression from pressures greater than those required to produce the above bulk thresholds. Using slower and often stepwise decompressions from these higher pressures, it was found that bubbles forming at and adhering to the glass-water interface were usually the first to appear; though often quite numerous $(20-200)$, they grew slowly in size. Upon continued decompression, bubbles appeared and developed in the bulk water.

The minimum gas supersaturation tension required for the onset of cavitation at the interface,

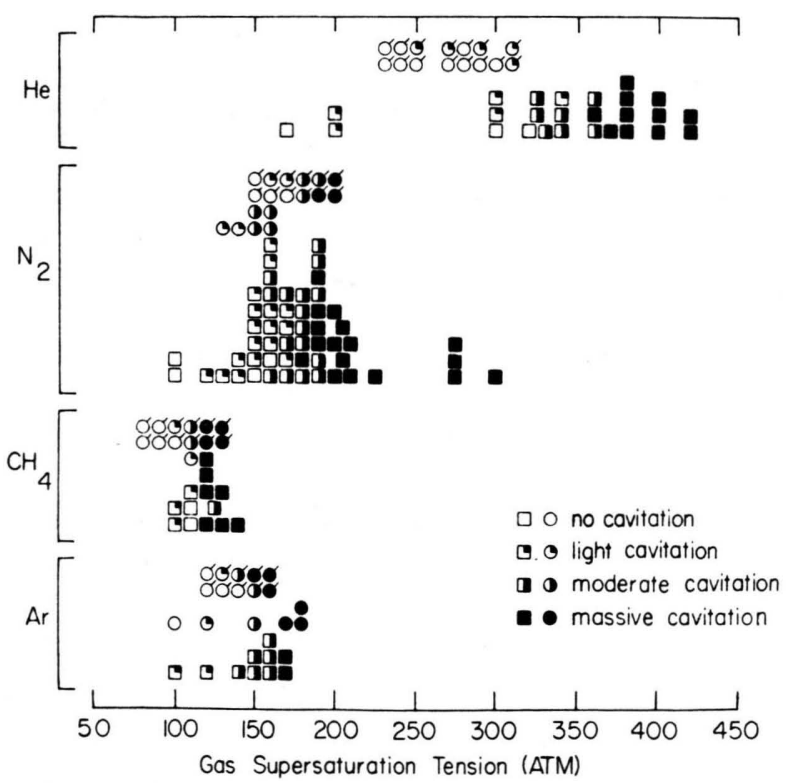

Fig. 2. Cavitation behavior of water supersaturated with various gases, decompressed to ambient pressure. The water was either hydrostatically pressurized to a minimum of 935 ATM for more than 15 minutes prior to equilibration with gas (squares), or was not pre-pressurized (circles). Tagged circles are representative data, reported in full elsewhere, obtained using a slightly different method ${ }^{3}$. Each symbol represents one experiment. i. e., the interface threshold, is shown as a function of gas equilibration pressure in Figure $3 \mathrm{~A}$. When decompressed from high gas equilibration pressures, the water tolerated lesser supersaturations before cavitating than when decompressed from lower gas equilibration pressures.

In Fig. $3 \mathrm{~B}$, the interface thresholds are normalized, for each of the gases respectively, about their bulk threshold at ambient pressure. Each normalized supersaturation $(F)$ is defined as:

$$
F=\left[\left(n \cdot P_{1}\right)-P_{\mathrm{c}}\right] / P_{1}
$$

where $P_{1}=$ the bulk threshold at ambient pressure; $n \cdot P_{1}=$ the gas equilibration pressure expressed in terms of $P_{1}$; and $P_{\mathrm{c}}=$ the hydrostatic pressure at which cavitation was first observed to occur during decompression from $n \cdot P_{1}$. The normalized curves indicate that the relative stability of all the gas solutions was affected similarly by increasing saturations. At first, the interface threshold decreased rapidly with increasing equilibration pressures, and then they tended to level off to a slower change at higher pressures. For example, the interface thresholds, with decompressions from $320 \mathrm{~atm}$, were nearly half those with decompressions from pressures required to produce the bulk thresholds at ambient pressure, and with decompressions from $550 \mathrm{~atm}$ gas saturations, they had dropped to about a third.

Even though the bulk thresholds were more difficult to quantify due to interference of water movement and gas depletion from the expansion of bubbles at the interface, varying the gas equilibration pressure appeared to have a differing effect upon the interface threshold relative to the corresponding effect upon the bulk threshold, depending upon the decompression rate. Thus, during slow gradual decompressions, bubbles occurred first at the interface at consistently lower supersaturations than those required for cavitation in bulk. In contrast, during very rapid decompressions, the appearance of these interfacial bubbles was delayed enough for decompression to proceed to the larger supersaturations required for nucleation in bulk water, which in these cases, was observed to occur either concurrently with or before the interfacial cavitation.

\section{Discussion}

Cavitation inception in gas supersaturated liquids may occur in the three different ways; inception (i) by pre-formed gas nuclei stabilized either on par- 


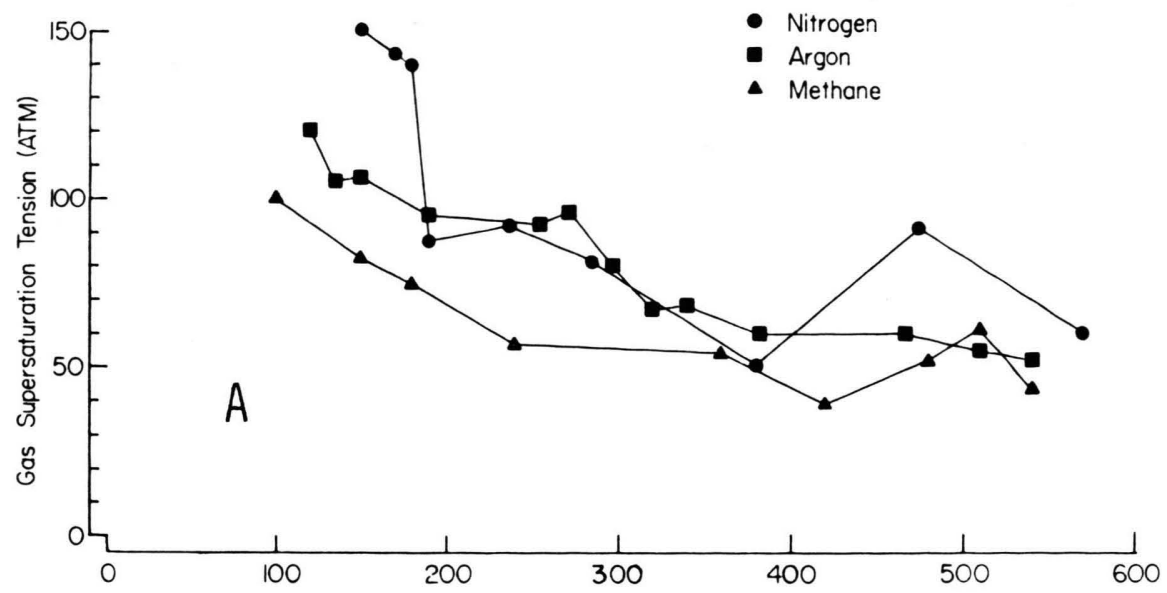

Fig. 3. A) Minimum gas supersaturation tension re quired for cavitation initiating at the glass-water interface as a function of the gas equilibration pressure $\left(n \cdot P_{1}\right)$. Each point represents the lowest such supersaturation tension obtained from several experiments using a given gas equilibration pressure.

B) Relation with gas equilibration pressure of the above interface threshold supersaturations, normalized about the respective bulk cavitation threshold supersaturation tension at ambient pressure $\left(P_{1}\right): \mathrm{CH}_{4}$, $P_{1}=120$ ATM $;$ Ar, $P_{1}=$ 160 ATM; $\mathrm{N}_{2}, P_{1}=190$

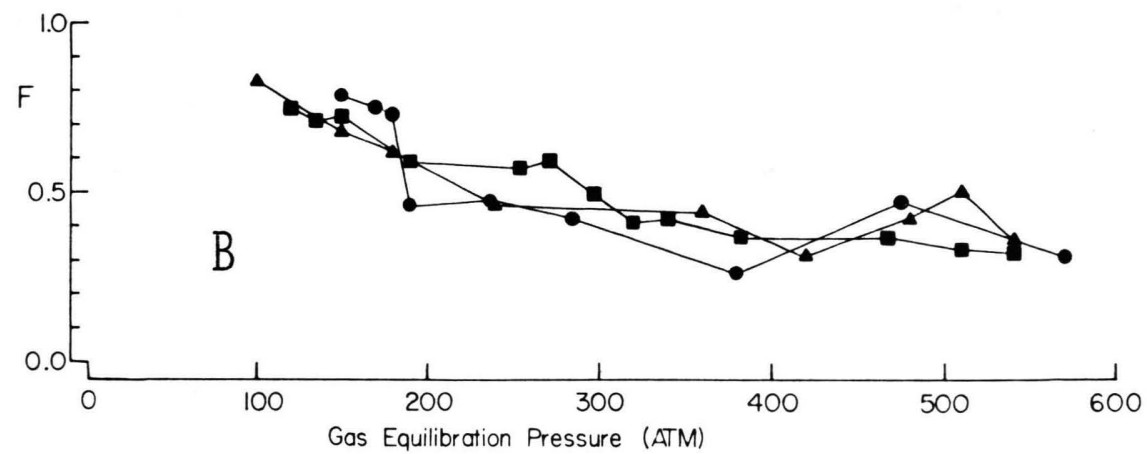
ATM.

ticles suspended in the liquid ${ }^{4,8-10}$ or on container surfaces $^{8}$, (ii) by nuclei formed spontaneously in bulk liquid, involving only dissolved gas and the liquid ${ }^{11}$, and (iii) by nuclei formed spontaneously at solid-liquid interfaces ${ }^{11-13}$.

In the present experiments, cavitation was initiated predominantly by the latter two processes. This is substantiated by several observations made here and in other related investigations ${ }^{3}$ which are inconsistent with the behavior expected for preformed gas nuclei. (I) The interface threshold supersaturations qualitatively paralleled the corresponding bulk threshold supersaturations; only moderate increases of supersaturation over those showing no cavitation were sufficient to elicit a dense uniform distribution of bubbles throughout the water volume and at the glass-water interface of the capillary. (II) Both interface and bulk thresholds were different for and characteristic of each gas. (III) Hydrostatic pre-pressurization of the water had no effect on the bulk thresholds and only rarely affected the interface thresholds. (IV) The interface thresholds were not constant, even for a given gas in water, but decreased with increasing gas equilibration pressure.

In some cases showing only very light cavitation, the contribution of pre-formed nuclei may have been significant and indeed, they may have been responsible for a few of the cases in which such light cavitation occurred at supersaturations unusually low with respect to that required to elicit massive cavitation. Generally, however, it appears that the application of gas pressure induces sufficient hydrostatic pressure in the water to force nearly all pre-formed nuclei into solution ${ }^{3}$. Thus, though threshold supersaturations remain relatively unaffected by preformed nuclei, the maximum supersaturation eliciting no cavitation may be sensitive to this type of interference, as are some other types of cavitation processes. For example, in determinations of the tensile strength of liquids even a single nucleation focus is sufficient to interrupt the cohesive continuity of the stressed liquid ${ }^{10,14-17}$.

Since our experimental technique only allowed quantitative determination of the thresholds for cavitation at the glass-water interface, many of the 
inferences made here apply strictly only to this type of spontaneous nucleation. However, inasmuch as bulk water cavitation required only moderately higher supersaturations, and in other respects did not appear to differ substantially from the interface cavitation, some general conclusions may be reached about the nucleation process.

The observation that bubbles can occur spontaneously in a given gas-liquid solution at different supersaturation tensions (Fig. 3) raises a question about the adequacy of the Laplace equation for estimating a critical size of the initial nuclei formed. This equation, conventionally applied to descriptions of the nucleation process ${ }^{8,18,19}$ gives the equilibrium critical radius $\left(r_{\mathrm{cr}}\right)$ of a spherical bubble above which growth to macroscopic size proceeds unabated, and below which collapse back into solution occurs. The minimum supersaturation tension $(\triangle P)$ in excess of any given hydrostatic pressure, required by an incipient nucleus to reach this critical size is given by $\Delta P=2 \sigma / r_{\mathrm{cr}}$, where $\sigma=$ the gas-liquid surface tension. This relationship when applied to the present data requires that either the surface tension or the critical radius, or both, varied as a function of pressure.

Several facts indicate that variation of the surface tension cannot account for our observations. Even though the surface tension of water varies with increasing gas partial pressure at the gas-water interface ${ }^{20}$, this variation over the ranges of gas pressures used here amounts to only a few percent, both with increasing gas partial pressure and between different gases ${ }^{20}$, whereas the observed reduction of the interface thresholds was, in each case, greater than 60 percent. It is unlikely that the nucleation event can be sensitive enough to these relatively small changes in surface tension to cause such large reductions of the interface threshold values. Furthermore, such sensitivity would cause the threshold curves for the various gases to diverge from each other with increasing gas saturations. This was clearly not the case; the relative threshold supersaturations varied similarly with increasing equilibration pressures. Previous data also discount such an extreme sensitivity to changes of surface tension; only minor variations of cavitation properties occurred in different liquids with wide ranges of surface tension ${ }^{3}$. Since neither the interface nor bulk thresholds can be related to known variations of surface tension, it is doubtful that this parameter imposes a major limitation on the nucleation process. Another point which must be mentioned here is the unreasonable critical size requirement which application of the Laplace equation imposes on nuclei ${ }^{3}$.

Rather, it appears that the total dissolved gas concentration is an important factor limiting the nucleation process. The threshold supersaturations generally are lower when the concentration of dissolved gas is higher. This applies to experiments where the gas concentration is varied in a given gaswater solution and to experiments in different liquids with various solubilities of various gases.

However, for each gas, the decrease in the interface threshold tends to level off to a slower change with progressively greater saturations. This and the abrupt drop in supersaturation stability observed in water below $3.5^{\circ} \mathrm{C}^{3}$, indicates that properties of the gas-liquid solution other than those related directly to gas concentration can also limit the minimum threshold values. Thus, the present observation that bubbles usually first appear at the glass-water interface must be related to interactions between the gas and solvent liquid which are peculiar to the boundary layer immediately adjacent to the interface. For example, gas adsorption or kinetic interactions at the interface, or variations in water structure induced by proximity to the interface ${ }^{27,28}$ or a flux anisotropy induced by the interface upon interfacial nuclei ${ }^{21}$ may become significant.

The mechanism by which gas concentration affects the nucleation process is not yet clear. Spontaneous nuclei have been suggested to arise from "holes" or density dilations postulated to occur uniformly in bulk liquid as a result of thermal fluctuations ${ }^{11,22-25}$. Successful nucleation requires that some of these nuclei reach and exceed some critical size ripidly enough to overcome the tendency for collapse and resolution. This may occur both by aggregation of nuclei and accumulation of gas from solution into the nuclei. Increasing gas concentrations may increase the temporal stability of these subcritical nuclei and/or their growth rates; each effect could augment the aggregation of such nuclei and facilitate successful nucleation at lower supersaturation tensions. Also, the solution of gases in water may induce changes in the molecular structure of the water ${ }^{26}$ which could in turn cause the gas supersaturation stability to vary with dissolved gas concentration. 
1 This work was supported by grant numbers HL 50263 and HL 16855 from the National Institutes of Health, U.S. Department of Health, Education and Welfare.

2 F. B. Kenrick, K. L. Wismer, and K. S. Wyatt, J. Phys. Chem. 28, 1308 [1924].

3 E. A. Hemmingsen, J. Appl. Phys. 46, 213 [1975].

4 E. N. Harvey, A. H. Whiteley, W. D. McElroy, D. C. Pease, and D. K. Barnes, J. Cell. Comp. Physiol. 24, 23 [1944].

5 P. B. Bennett and D. H. Elliott, eds., The Physiology and Medicine of Diving and Compressed Air Work, Williams and Wilkins, Baltimore 1975.

6 C. J. Lambertsen, ed., Underwater Physiology, Academic Press, New York 1971.

7 R. J. R. Johnson, G. Froese, M. Khodadad, and D. Gibson, Br. J. Radiol. 41, 749 [1968].

8 E. N. Harvey, D. K. Barnes, W. D. McElroy, A. H. Whiteley, D. C. Pease, and K. W. Cooper, J. Cell. Comp. Physiol. 24, 1 [1944].

9 F. E. Fox and K. E. Herzfeld, J. Acoust. Soc. Amer. 26, 984 [1954].

10 M. S. Plesset, O. N. R. Report No. 85-47, 1969.

11 R. Fürth, Proc. Camb. Phil. Soc. Math. Phys. Sci. 37, 276 [1941].

12 D. C. Pease and L. R. Blinks, J. Phys. Colloid Chem. 51, 556 [1947].
13 D. J. Cotton, Phil. Mag. 25, 77 [1972].

14 R. E. Apfel, Sci. Amer. 227, 58 [1972].

15 R. E. Apfel, J. Acoust. Soc. Amer. 48, 1179 [1970].

16 J. W. Holl, J. Basic Eng. 92, 681 [1970].

17 M. Strasberg, J. Acoust. Soc. Amer. 31, 163 [1959].

18 J. P. Hirth and G. M. Pound, Condensation and Evaporation: Nucleation and Growth Kinetics, Pergamon Press, The MacMillan Corp., New York 1963, pp. 149 $-160$.

19 C. A. Ward, Univ. of Toronto Tech. Pub. Ser., U. T. Mech. E. TP 7105 [1975].

20 J. C. Eriksson, Acta Chem. Scand. 16, 2199 [1962].

'21 W. M. Buell and J. W. Westwater, American Institute of Chemical Engineers Journal 12, 571 [1966].

2.2 R. Fürth, Proc. Camb. Phil. Soc. Math. Phys. Sci. 37, 252 [1941].

23 W. Döring, Z. Phys. Chem. B 36, 371 [1937] and ibid. B 38, 292 [1938].

24 J. C. Fisher, J. Appl. Phys. 19, 1062 [1948].

25 R. D. Finch, Phys. Fluids 12, 1775 [1969].

26 A. Ben-Naim, J. Chem. Phys. 45, 2706 [1966].

27 W. Drost-Hansen, in Chemistry of the Cell Interface, edited by H. D. Brown, Academic Press, New York 1971, pp. $1-184$.

28 R. D. Schultz and S. K. Asunamaa, Rec. Progr. Surf. Sci. 3, 291 [1970]. 